\title{
Les Roms migrants en région parisienne : les dispositifs d'une marginalisation
}

Roma Migrants in the Paris Suburbs: Mechanisms of Marginalization

Los migrantes Roma en la region de Paris: los mecanismos de la marginalizacion

\section{Alexandra Nacu}

\section{(2) OpenEdition}

\section{Journals}

Édition électronique

URL : https://journals.openedition.org/remi/5047

DOI : $10.4000 /$ remi. 5047

ISSN : $1777-5418$

Éditeur

Université de Poitiers

Édition imprimée

Date de publication : 1 février 2010

Pagination : 141-160

ISBN : 978-2-911627-54-5

ISSN : 0765-0752

Référence électronique

Alexandra $\mathrm{Nacu}$, «Les Roms migrants en région parisienne : les dispositifs d'une marginalisation », Revue européenne des migrations internationales [En ligne], vol. 26 - $n^{\circ} 1$ | 2010, mis en ligne le 01 février 2013, consulté le 15 avril 2022. URL : http://journals.openedition.org/remi/5047 ; DOI : https://doi.org/ 10.4000/remi.5047 


\title{
Les Roms migrants en région parisienne : les dispositifs d'une marginalisation
}

\author{
Alexandra NACU*
}

S'inscrivant de manière particulièrement visible dans le paysage urbain, les migrations des Roms focalisent de manière croissante les discours populistes et l'attention médiatique. Ces migrants, originaires surtout de Roumanie et de Bulgarie mais aussi d'autres pays des Balkans font périodiquement l'objet d'un traitement sensationnaliste de la part des médias français, mais aussi de ceux des pays de départ. Ils sont présents en France pour certains depuis les années 1990 sans que leur situation change durablement, malgré les multiples évolutions juridiques caractérisant cette période.

Cette recherche s'intéresse aux Roms est-européens installés en banlieue parisienne et aux formes d'intervention institutionnelle (pouvoirs publics et ONG) à leur endroit. En analysant ce phénomène à différentes échelles (européenne et locale), je montre comment ce groupe, sédentaire à l'origine, est maintenu avec le concours de l'action des pouvoirs publics dans un nomadisme forcé, perpétuant et même renforçant ainsi sa marginalisation. Cet article montre de quelle manière ces habitants au statut juridique ambigu, bénéficiant du droit à la présence sur le sol français depuis janvier 2007 mais privés du droit au travail, font l'objet d'une gestion plus ou moins identifiable et cohérente, souvent intermittente et « par défaut».

Le terrain de cette recherche a associé d'une part, des entretiens approfondis avec les migrants, les responsables politiques et sanitaires locaux, les ONG intervenant auprès de cette population, et d'autre part, des périodes prolongées d'observation participante avec l'association Médecins du Monde (Mission Banlieue). La majeure partie des observations et entretiens datent des années 2004-2006, mais la recherche s'est poursuivie jusqu'en 2009 pour prendre en compte les effets de l'aprèsélargissement de l'UE.

* Post-doctorante au Centre de Sociologie des Organisations (CNRS - Sciences-Po), 13, rue de l’Université 75007 Paris, alexandra.nacu@sciences-po.org 
L'intérêt académique pour les migrations des Roms n'a pas, jusqu'à présent, suivi l'intérêt médiatique. Alors que de nombreuses recherches portent sur la marginalisation des Roms en Europe de l'Est (Stewart, 1997) et sur les discriminations dont ils font l'objet (Gugliemo, 2004 ; Commission Européenne, 2005), leurs migrations vers l'Ouest n'ont pas fait l'objet d'une attention scientifique aussi soutenue. Qui plus est, du fait d'une apparente singularité du mode de vie des Roms et de certaines de leurs activités on a eu tendance à mettre leur migration en contraste avec celle des non-Roms (Castle-Karenova, 2003 ; Diminescu et Lagrave 1999). Nous verrons dans cet article de quelle manière les migrations des Roms, bien que perçues sous le signe de l'altérité, partagent bien des attributs avec celles des autres est-européens. Ces similitudes interrogent le chercheur sur les raisons du maintien de cette population dans la marginalité extrême où elle se trouve.

Plusieurs paramètres législatifs, institutionnels et politiques affectent et limitent ces migrations : les régimes d'entrée et de séjour, mais aussi les pratiques de la police et la politique nationale et locale en matière de tolérance/expulsion des irréguliers, des occupations de terrains publics ou privés, du travail informel ou de la mendicité. Je m'intéresse à cet encadrement et à ses conséquences, d'abord au niveau européen et national, puis, par ses effets sur l'organisation interne des migrations, enfin, dans les diverses politiques locales à l'égard des groupes de Roms migrants.

\section{S'ADAPTER À L'ÉVOLUTION DES DISPOSITIFS JURIDIQUES}

Souvent interprétés à tort comme une manifestation de la nature nomade des Roms ${ }^{1}$, ces mouvements de populations sont en partie des migrations de travail ratées, les travailleurs potentiels échouant à intégrer les circuits de travail illégal et recourant à la mendicité comme une alternative viable. Comme nous le verrons, c'est la position très basse sur l'échelle sociale dans le pays d'origine qui explique à la fois cet échec (dû au manque de capital social qui leur permettrait d'intégrer ces réseaux) et la relative facilité avec laquelle ils adoptent la solution de la mendicité, en raison d'une forme spécifique de capital social ${ }^{2}$. Cependant, il ne faut pas minimiser la difficulté d'adaptation à ce statut, qui transparaît dans les entretiens cités ci-dessous et qui est indissociablement liée à la condition du mendiant.

Il s'agit de migrations circulaires entre la région d'origine et Paris, les migrants demeurant en France pour une durée de quelques mois et revenant pour des périodes généralement plus brèves dans leur région d'origine. Le nombre des migrants est par définition difficile à évaluer, en raison du caractère informel et temporaire de l'habitat, des mouvements incessants entre squats et bidonvilles et des allers-retours au

1 La sédentarisation de parties importantes des groupes de Roms est une réalité ancienne, remontant au-delà du XIX ${ }^{\text {ème }}$ siècle. Les régimes communistes poussèrent plus loin ces efforts de sédentarisation jusqu'à comprendre la quasi-totalité des Roms est-européens (Crowe, 2007).

2 Même si ces migrants sont en majorité des Roms, j'ai pu au cours de ma recherche rencontrer une minorité significative de non-roms, dont quelques-uns habitaient sur les mêmes terrains que les Roms. 
pays d'origine. Selon un rapport de l'organisation Romeurope, la population des bidonvilles roms roumains et bulgares atteint quelques milliers en France, dont quelque 3000 en Ile-de-France (Romeurope, 2008). En 2005, l'association Médecins du Monde (MDM) avait recensé environ 2700 Roms vivant dans des bidonvilles et des squats en Ile-de-France, mais ce chiffre prend uniquement en compte les lieux connus de MDM, c'est-à-dire les bidonvilles et squats les plus importants. Sans doute cette population est-elle plus nombreuse si l'on inclut les petits « terrains » ou les squats.

\section{Des migrations sous le signe de l’UE : une lente évolution}

Nouveauté de l'après-1989, survenant après des décennies de fermeture des frontières, la migration vers l'Europe occidentale de parties significatives de la population est devenue un fait social d'importance majeure en Roumanie et en Bulgarie, tant pour ceux qui partent que pour ceux qui restent. Les migrations circulaires de travail (formel ou informel) y prennent une place importante (Potot, 2007).

Les modalités de ces migrations ont évolué avec les changements politiques et juridiques. Pendant les années 1990, les pratiques nationales d'octroi de l'asile politique ont joué un rôle important dans ces mouvements de population. Les Roms, comme la plupart des migrants roumains et bulgares à cette époque passaient par la demande d'asile pour bénéficier au moins d'une période initiale de séjour légal en France. Cependant, à la fin des années 1990, les demandes d'asile de la part des Roms roumains ou bulgares sont devenues exceptionnelles, la France comme les autres pays européens pratiquant la politique plus ou moins explicite des « pays sûrs ». Pour les ressortissants de ces pays, la question s'est posée de savoir si les migrations des Roms relèvent de la problématique de l'asile politique (Vasecka et Vasecka, 2003). En réalité, la question : «sont-ce de véritables réfugiés ou des migrants économiques ? » se pose pour beaucoup de demandeurs d'asile. Dans le cas des Roms les deux aspects, discrimination et statut économique dans les pays d'origine, sont intimement liés (Gugliemo, 2004).

À partir des années 2000 et jusqu'à l'intégration à l'UE de la Roumanie et de la Bulgarie en 2007, comme cela avait été le cas pour les pays d'Europe Centrale avant leur entrée dans l'UE, les Roms migrants de ces deux pays ont constitué un objet de négociation entre la Roumanie, la Bulgarie et l'UE. C'était là une question épineuse pour les pays d'origine dans le processus d'accession à l'UE, d'autant qu'il s'agissait aussi pour ces pays de définir une identité nationale qui ne soit pas indissociablement liée à la pauvreté (Nacu, 2006).

La plupart des Roms est-européens présents aujourd'hui en région parisienne sont arrivés depuis le début de la décennie 2000, mouvement facilité par la suppression (en 2001 pour la Bulgarie et en 2002 pour la Roumanie) des visas d'entrée dans l'espace Schengen pour la Roumanie et la Bulgarie (ouvrant le droit à un séjour de trois mois en tant que touriste, à condition de faire la preuve de disposer de certaines ressources à la sortie du pays d'origine). Ceci a également renforcé la circularité de la migration. 
Cela ne signifie pas que le statut de ces migrants était toujours légal. Avant 2007, la plupart dépassaient le délai légal de 3 mois de présence dans l'espace Schengen, retournant par la suite dans leur pays pour «nettoyer » leur passeport (terme utilisé en roumain et en bulgare). D'autre part, même au cours des 3 mois autorisés, ceux-ci étaient passibles de reconduite à la frontière parce qu'ils ne correspondaient pas à la notion de « touriste » (celui-ci est censé pouvoir subvenir à ses besoins et donc, par exemple, ne pas faire la manche).

Une tension est apparue dans le processus d'accession européenne des deux pays : d'une part, l'UE promouvait, de manière explicite, l'égalité des droits des Roms est-européens dans ces pays et émettait régulièrement des avertissements à l'adresse de ces derniers, menaçant de retarder leur adhésion en raison de la non-satisfaction de ces conditions. D'autre part, tout au long des pourparlers d'adhésion, la plupart des États membres ont maintenu une pression sur ces pays pour qu'ils retiennent les Roms et les autres immigrés indésirables à l'intérieur de leurs frontières, en niant par là leur égalité de droits avec les autres citoyens. La dénonciation des violations des droits de l'homme dans les pays candidats était d'ailleurs une position à double tranchant pour les États de l'UE, car ceci équivalait à justifier les demandes d'asile. C'est pourquoi le langage des droits de l'homme a progressivement cédé la place aux discours sur les inégalités sociales chez les rapporteurs de l'UE (Castle-Karenova, 2003).

Cette période correspond à un « contrôle à distance » (Bigo et Guild, 2003) délégué aux pays de départ. Les mesures de contrôle et de tri des migrants, de sanction des infractions au droit du séjour, revenaient notamment aux pays de départ, auxquels on a donné l'essentiel du rôle de répression. Lorsqu'une personne dépassait les trois mois de séjour réglementaires, non seulement le séjour dans l'espace Schengen mais aussi le retour au pays posaient problème, car la police des frontières du pays d'origine pouvait alors confisquer son passeport ou émettre une interdiction de sortie du territoire comprise entre plusieurs mois et plusieurs années. Cependant, ce dispositif était loin d'être étanche. Aussi cette contradiction inhérente dans les orientations des politiques migratoires s'est-elle traduite pendant cette période par une grande place laissée à l'arbitraire aux frontières. Ces contraintes ont cependant été compensées par divers mécanismes de corruption monétaire aux frontières des pays de départ, qui a fait office de variable d'ajustement dans la régulation des mouvements de personnes, situation pénalisante pour les plus pauvres, ceux qui ne disposent pas des moyens financiers ou des renseignements permettant un passage réussi de la frontière.

Aussi, selon les entretiens avec les migrants, pouvait-on rentrer au pays moyennant un paiement informel à la frontière, mais, si cette solution était généralement sans problème particulier, elle pouvait comporter une part de risque : un « super contrôle » (lorsque les policiers de frontière sont contrôlés par un émissaire de la hiérarchie) inopiné à la frontière, ou un douanier plus exigeant ou plus scrupuleux que la norme risquaient de remettre en question l'entreprise de subornation ${ }^{3}$. Les Roms

3 Ce problème s'est posé de façon spectaculaire pendant l'été 2005 en Roumanie, lorsque, suite à un durcissement subit des règles, en trois jours, plusieurs milliers de retardataires ont été 
Bulgares de la région parisienne rentraient souvent via Istambul, par un vol des compagnies aériennes bon marché, d'où ils empruntaient un bus pour rentrer en Bulgarie. D'après leurs déclarations, les gardes-frontières de la frontière turque «ne demandent même pas de bakchich » (Entretien, Rom bulgare, décembre 2005).

Soulignons que les inégalités sociales et ethniques jouaient fortement dans cet aspect du « contrôle à distance » et donc dans l'accès à la mobilité internationale. La pratique des pots-de-vin œuvre en défaveur des plus pauvres. Loin d'être réduits à la même condition par le fait de se rendre à l'étranger, la discrimination sur critères ethniques se manifestait là aussi, que ce soit à la frontière, lorsque les Roms étaient plus souvent sollicités pour des pots-de-vin, comme de nombreux entretiens avec des migrants Roms et non-Roms me l'ont fait comprendre, ou dans les rues des villes occidentales, où ils étaient davantage susceptibles d'être interpellés dans le cadre de « contrôles au faciès ». Mais ces inégalités dans la mobilité sont aussi ressenties au niveau du pays de départ tout entier, dans la question de l'accès inégal à la libre circulation et au droit de travailler en fonction du pays dont on est le ressortissant : la question des accès différenciés des pays est-européens à l'Occident sans visa est un sujet de prédilection de la presse nationale des deux pays et un thème électoral important.

L'intégration des deux pays dans l'Union Européenne en janvier 2007 a mis fin à ces pratiques aux frontières des pays de départ. Les citoyens des deux nouveaux États membres ont, en principe, le droit de résider en France pour une durée de 3 mois. Cependant, un régime dérogatoire transitoire (qui peut être prolongé jusqu'en 2014) leur est appliqué, imposant notamment l'obligation d'obtenir une autorisation de travail pour être embauchés ${ }^{4}$. De plus, une circulaire stipule que les Roumains et les Bulgares présents sur le territoire français ne doivent pas représenter une « charge déraisonnable pour le système d'assistance sociale français $»^{5}$, notion assez large qui rend les Roms passibles d'une amende et de l'expulsion lors d'un contrôle policier dans la rue. C'est en invoquant cette disposition que de nombreuses OQTF (Obligation de Quitter le Territoire Français) ont été émises à partir de 2007 à l'encontre de Roms roumains et

interpellés à la frontière entre la Hongrie et la Roumanie, et leurs passeports confisqués. Le retard, qui auparavant pouvait se régler avec un paiement de 50 euros glissés dans le passeport $\mathrm{du}$ retardataire était subitement devenu une infraction punie par la loi. La raison de cette observance exceptionnelle de la loi réside dans une décision du président Basescu, suite à une requête directe de l'UE qui menaçait, une fois de plus, d'ajourner l'entrée de la Roumanie dans l'UE si celle-ci ne renforçait pas le contrôle sur les migrants (voir « Bucarest durcit le contrôle aux frontières pour ses propres ressortissants », Le Monde, 10/08/05). Cette situation soudaine a été ressentie comme une injustice criante par les intéressés («50, d'accord c'était normal, mais 300, ça dépasse les bornes », entretien avec un Rom roumain, Paris, septembre 2005). Environ six mois après cet incident, d'après les déclarations des migrants, le cours des paiements informels était retombé aux anciens prix.

4 http://www.servicepubliclocal.com/spl/accueil.spl?c=547706\&f=N0/N9/N123/N13510/N13524/F13526/, dernier accès : mai 2009.

5 Circulaire INT/D/06/000115/C du 22 décembre 2006 (ministère de l'intérieur) relative aux modalités d'admission au séjour et d'éloignement des ressortissants roumains et bulgares à partir du $1^{\mathrm{er}}$ janvier 2007. 
bulgares, certaines suivies de vols de retour groupés organisés par l'ANAEM (Agence Nationale d'Accueil des Étrangers et des Migrations), devenue en 2009 l'OFII (Office Français d'Immigration et de l'Intégration) ${ }^{6}$.

Depuis janvier 2007, les Roumains et les Bulgares ont donc en principe le droit de travailler en France dans 62 professions qui connaissent une pénurie de maind'œuvre. Cependant, obtenir l'autorisation de travail est une procédure longue et incertaine qui demande au futur employeur de s'acquitter d'une taxe d'environ 900 euros et d'attendre environ deux mois pour connaître la réponse à sa demande. L'on comprend que peu d'employeurs soient prêts à s'engager dans de telles démarches, qui introduisent une inégalité supplémentaire entre migrants ; d'après les entretiens, le peu de cas où celles-ci aboutirent concernaient des migrants non Roms qui avaient depuis longtemps noué des liens avec leurs employeurs. Ces contraintes font que l'intégration européenne a jusqu'à présent peu modifié les conditions de vie des Roms migrants, si ce n'est le passage d'un contrôle international de leur migration à un contrôle de plus en plus local.

\section{Une mobilité croissante malgré les obstacles}

À la différence des migrants non-européens, le retour en France a toujours été beaucoup plus aisé pour les migrants roumains et bulgares, en raison de la porosité des contrôles aux frontières décrits ci-dessus. On peut se demander quel est le sens des mesures d'éloignement et d'expulsion de cette population qui peut à présent revenir en France beaucoup plus aisément, ce que la plupart des expulsés ne manquent pas de faire quelques semaines après la mesure ? On peut penser que l'expulsion de nationaux roumains et bulgares, roms notamment, a une fonction d'illustration - voire de communication politique - d'une politique de l'immigration. Ainsi, il arrivait régulièrement avant 2007 que des personnes en situation irrégulière soient arrêtées puis relâchées car les circuits institutionnels de l'expulsion étaient engorgés par manque de personnel ${ }^{7}$.

Il en est de même des programmes de « retour aidé ${ }^{8}$. Les accords signés en 2004 par le ministre de l'intérieur Nicolas Sarkozy avec les gouvernements roumain et bulgare pour le retour et la réintégration des migrants roms n'ont pas abouti au retour définitif de ces migrants (FIDH, MDM, 2003). Le détournement des programmes de retour aidé de l'ANAEM/OFII relève les mêmes ambiguïtés : les migrants, dans leur immense majorité, ne souhaitent pas «être réintégrés dans leur pays », où ils voient le plus souvent une impasse économique, mais regagner l'Occident le plus tôt possible, ce

6 Voir par exemple l'article "Les "retours humanitaires" de Roms se sont accélérés », in Le Monde, 30/12/2007.

7 « On ne peut plus augmenter le nombre de reconduites », 20 minutes, 24/01/06.

8 L'ANAEM offrait aux migrants qui souhaitaient rentrer de leur plein gré un billet d'avion et un pécule (de 150 euros environ en France en 2006-2007, celui-ci fut porté à 300 euros en 2008). Voir la dépêche AFP "Un collectif accuse Brice Hortefeux "d'acheter" des retours parmi les Roms », 07/04/08. http://afp.google.com/article/ALeqM5iFLsQyJJp_vVL0iCeJGGKJQdKHw?index=0 dernier accès mai 2009. 
qu'ils ne tardent pas à faire. La politique des éloignements a pour effet de fragiliser leur statut - ils sont passibles d'amende ou d'emprisonnement s'ils ne quittent pas la France dans les délais stipulés - mais ne parvient pas à les éloigner durablement du territoire français.

Cependant, cette politique de l'immigration contribue à maintenir cette population dans un statut juridique fluctuant, à géométrie variable. Si la menace de l'expulsion ne signifiait pas avant 2007 pour un Rom roumain ou bulgare la fin définitive de son séjour en France, elle représentait une fragilisation de ses perspectives : en permanence sous la menace d'amende, d'éloignement donc de rupture, fût-elle temporaire, des liens présents (famille, logement, activité), les Roms se voient confinés à la précarité. La plupart des éloignements dont j'ai été témoin ou dont j'ai eu connaissance au cours de mon enquête pendant la période 2004-2009 étaient collectifs, concernant la population d'un ou plusieurs terrains. Ils étaient organisés en coordination avec la police, les municipalités et suivis d'expulsion et ratissage des terrains par les bulldozers, donc d'une perte de leur habitat pour les Roms qui, de retour en France quelques semaines après pour la plupart, se mettaient à la recherche d'un nouvel emplacement de bidonville.

Par la facilité relative d'accès à l'Occident, ces migrations diffèrent des migrations africaines ou asiatiques, où les migrants doivent franchir de larges distances et de multiples frontières. Le prix du billet de bus Sofia-Paris ou Bucarest-Paris était en 2007 de 150 euros aller-retour. De nombreux migrants utilisent des minibus privés, pratiquant des prix inférieurs pour faire le trajet directement depuis leur ville d'origine. Même lorsqu'une condition financière d'entrée dans l'espace Schengen existait, il était possible à presque tout un chacun d'emprunter 200 euros afin de pouvoir payer le trajet ou les pots-de-vin aux frontières (ce qui se faisait au moyen d'une collecte dans le bus, le chauffeur gérant les relations avec les gardes-frontière (Entretien avec des Roms roumains, mars 2004).

Les migrants utilisent ainsi les failles et les niches des différents dispositifs juridiques et administratifs nationaux. Diverses autres conditions affectent les migrations : la possibilité de squatter un immeuble ou de faire la manche diffère de pays à pays. Par exemple, en France, une loi d'août 2003 interdisant la mendicité avec un enfant de moins de 6 ans a fortement enrayé cette pratique. Les migrants communiquent entre eux sur les différentes possibilités qui leur sont ouvertes. Ceux-ci ne disposent pas toujours des renseignements fiables sur les pays d'immigration, mais des informations circulent et sont rapidement relayées. La grande mobilité est accentuée par l'attirail technologique de la mondialisation : téléphones portables que de nombreux migrants possèdent, envoi rapide d'argent par Western Union, capacité à regarder les informations télévisées même dans un bidonville. Dans l'extrait suivant, un rom bulgare résume sa vision de ce que l'Europe peut lui offrir :

"L'Allemagne, n'y pense même pas, ils ne te laisseront jamais entrer dans une maison [pour la squatter], ni faire la manche. La Belgique, maintenant c'est très dur, il faut y aller uniquement si tu veux rentrer par l'OMI'. Oui, [rires] les Belges

9 L'Organisation Internationale pour les Migrations, est partenaire des gouvernements pour les programmes de retour aidé. En 2004, selon eux, la section belge de l'OMI était connue par les Roms bulgares de Paris comme facilitant le retour car moins bureaucratique, pratiquant moins le tri parmi les migrants. 
ils en ont tellement marre de nous qu'ils te paient ton billet retour illico, c'est pour ça que tout le monde va chez eux. L'Espagne c'est bien d'après ce qu'on dit, certains des nôtres ont eu leurs papiers cet été. [Mais pourquoi n'y vas-tu pas ?] Ben à chaque fois que j' ai demandé aux nôtres qui sont là-bas, ils m' ont dit ' $y$ 'a pas de place". Je ne pouvais pas débarquer comme ça. » (Entretien avec un mendiant rom bulgare, Paris, décembre 2005).

Cet entretien reflète les informations dont un migrant disposait à un moment donné. Il suggère le fait que des conditions plus favorables dans un pays peuvent induire un flux de migrants dont la mobilité est très grande, mais que celle-ci est aussi conditionnée par les réseaux de migrants préexistants.

En France, la mendicité est l'activité de référence de ces personnes, celle qui, pour beaucoup, assure le meilleur rapport bénéfice/risque. Elle est tolérée de façon sélective selon le quartier et l'emplacement. Cette tolérance informelle joue un grand rôle dans les différentes possibilités qui s'ouvrent ou se ferment aux mendiants. Pouvoir faire la manche à tel endroit dépend de la bonne volonté des employés ou propriétaires des commerces devant lesquels les mendiants s'installent, des relations avec les policiers qui font leurs tours de garde. Certains quartiers, notamment les nouveaux quartiers « bobos » comme Oberkampf, Ménilmontant, sont particulièrement attractifs. Les quartiers touristiques classiques présentent une situation plus complexe : théoriquement très prisés car lucratifs, certains sont inabordables en raison de la répression policière ou obligent les Roms à adopter des stratégies de mendicité plus mobiles (« à la tour Eiffel, on ne nous a jamais laissés nous asseoir [pour faire la manche] », (migrant bulgare, Paris, juin 2005).

\section{L'INSTALLATION EN FRANCE}

\section{Des réseaux faiblement structurés}

Le type d'activité pratiqué par ces personnes est essentiellement la mendicité et/ou des activités similaires ${ }^{10}$, comme la collecte de métaux recherchés par les centres de recyclage ${ }^{11}$, la vente de fleurs, de journaux pour les sans-abri, ou la pratique de la musique dans la rue ou le métro, quelquefois le vol ou la prostitution. Parmi certains de ces migrants, il existe une grande continuité entre mendicité, petite délinquance et petits boulots au noir. Il s'agit, pour l'immense majorité, de personnes qui n'avaient jamais pratiqué la mendicité dans les pays d'origine, ce qui pose la question de l'installation dans ce type d'activité, donc de la «carrière » du mendiant (Damon, 1998). En

10 Diminescu et Lagrave (1999) parlent d' « entreprise de collecte » pour renvoyer de manière non-stigmatisante à cet ensemble d'activités, légales ou non (mendicité pillage des horodateurs).

11 Presque chaque terrain présente dans ses environs des résidus de feux allumés pour brûler l'isolation de câbles collectés afin de récupérer le fil de cuivre et le revendre (activité plus rémunératrice depuis l'augmentation considérable du prix de ce métal en raison de la demande chinoise). Cette activité implique souvent le vol de ces matériaux, comportant alors un grand risque. Elle est également pratiquée, de manière bien plus répandue, dans les pays d'origine. 
partant, beaucoup savaient qu'ils ne trouveraient pas de travail. D'autres espéraient vaguement une embauche. Mais au début des années 2000, ces nouveaux migrants se retrouvent dans une situation où le marché du travail informel est saturé, où trouver un emploi, même temporaire et mal rémunéré est conditionné par l'inscription dans des réseaux pré-existants. Ils vont à leur tour construire leur réseau de migrants, mais celuici les maintient dans la mendicité.

"En arrivant en France j" ai essayé de trouver du travail, pendant deux mois

j' arrivais pas à me décider à faire la manche. Il y a des Serbes, des Gitans français, qui te prennent à la journée, [pour travailler dans le bâtiment]. Ils m’ont promis de me payer, à chaque fois au bout d' une semaine je ne voyais rien venir, ou alors ils me donnaient 10, 20 euros. J'ai laissé tomber.

[Et comment tu as fait pour trouver du travail lors des vendanges?] J'ai pris le train, je suis arrivé à Belleville [Sur-Saône] [conseillé par une connaissance du quartier où il fait la manche], et là il y avait beaucoup de monde autour de la gare, et je vois un homme qui s'approche de moi. Je lui dis, "je cherche du travail", il me dit "d'accord mais as-tu des papiers?" Là je me tais. Il a compris que je n'avais pas le droit de travailler. Et j' ai su à quoi il pensait : tout de suite dans sa tête il a baissé le prix. [...]

Cette année j'y suis retourné, mais je n' ai pas pu travailler pour lui, il avait trop peur des contrôles, et il m' a refilé à son cousin qui est quelqu' un de pas bien.

Il ne me payait pas, et le travail était très dur. Au bout de 3 semaines, je suis revenu à Paris, je n' avais rien gagné avec tout ça, j' avais même perdu de l'argent. » (Entretien avec un migrant rom bulgare, Paris, octobre 2004)

La structure de ces activités a évolué depuis le début des années 1990. D’après les entretiens avec des travailleurs sociaux ayant une expérience de plusieurs années des populations roms, le nombre des mendiants a augmenté, et les phénomènes de réseaux mafieux de mendicité organisée ont régressé en comparaison avec les années 1990. Avec la suppression des visas, les flux de mendiants se sont transformés, et ces migrations sont devenues de plus en plus des entreprises individuelles ou familiales. À présent, la possibilité de migrer est ouverte à un très large nombre d'individus, qui, même sans disposer d'un revenu personnel, peuvent contracter des prêts qu'ils rembourseront après quelques mois de travail.

De façon similaire à d'autres migrations de travail provenant des Balkans, ces migrations sont spontanément constituées en réseau : quelques «pionniers » migrent vers une région et, après quelques mois, lorsque la migration commence à faire ses preuves, mesurées par l'argent envoyé au pays, d'autres les suivent. Cette notion de « réseau » est à entendre dans un sens large, celui d'une organisation informelle et mouvante constituée de liens lâches, définie ainsi par Douglas Massey : "L'ensemble des liens interpersonnels qui lient les migrants, les anciens migrants, et les nonmigrants dans les zones d'origine et de destination à travers les liens de parenté, amitié, et origine communautaire partagée » (Massey, 1998 : 396).

Cette forme du réseau explique le caractère en apparence très contingent et aléatoire de ces migrations : en effet, dans chaque ville occidentale on retrouve une surreprésentation de migrants provenant de certaines régions des pays de départ. De façon symétrique, dans les pays est-européens on rencontre, selon la région, des configurations très différentes qu'on ne peut pas toujours expliquer par leurs caractéristiques 
socio-économiques : on y trouve en effet des villages ou villes où pratiquement chaque famille a « quelqu'un à l'étranger », à côté d'autres lieux où la pratique de la migration est beaucoup moins répandue. Ceci n'est pas explicable principalement par les différences entre ces populations mais plutôt par des migrations qui ont pris leur origine dans un très petit noyau d'individus. Le réseau explique également le caractère paradoxal de la décision de migrer chez nombre de ces personnes : souvent déclarant partir « sur un coup de tête », sans préparation, ces migrants vivent cependant dans des sociétés où la migration est une possibilité toujours ouverte.

Après la mise en place du réseau, on observe un phénomène général dans les migrations internationales, explicité par Massey (1988), à savoir que la logique du réseau devient prépondérante et que celui-ci se nourrit de lui-même. À partir d'un certain seuil, d'un nombre suffisamment important de migrants, on observe une indépendance du processus de migration par rapport aux causes qui l'ont généré.

\section{Formation d'un bidonville : un regroupement sous contrainte}

L'une des spécificités de la migration des Roms est le type d'habitat auquel elle donne lieu. Alors que certains s'installent dans des squats allant jusqu'à quelques dizaines de personnes, d'autres bâtissent de véritables bidonvilles, appelés par les migrants «platz » en roumain (mot à consonance germanique héritage de premières migrations en Allemagne dans les années 1990), «palatki » (tentes, cabanes) en bulgare, et « terrains » dans le jargon des ONG humanitaires ${ }^{12}$.

Photo 1 : «Terrain» de Roms roumains sous un échangeur d'autoroute en banlieue Nord (A. Nacu)

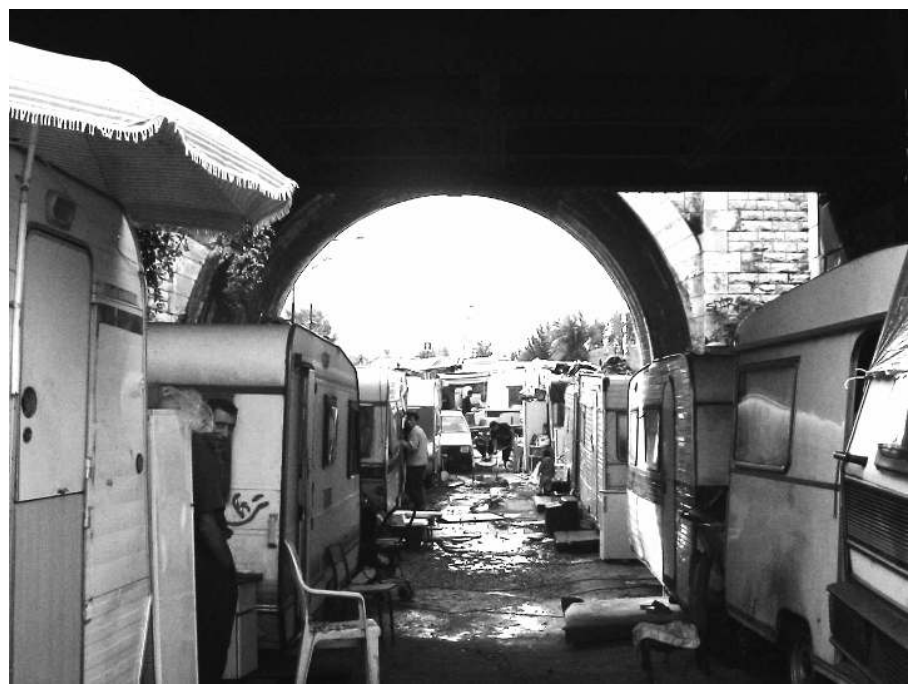

12 Sur les terrains que j'ai pu fréquenter, les organisations les plus présentes étaient MDM, Emmaüs, ATD Quart Monde, ASAV (L'association de défense des gens du voyage), le Comité d'Aide Médicale et divers comités de soutien (ceux de St-Denis, Bagnolet, Montreuil, St-Michel-sur-Orge étant les plus actifs). On y rencontre également des citoyens agissant à titre personnel. 
Les « terrains » sont des agglomérations constituées de caravanes de deuxième main ou de cabanes en bois ou d'autres matériaux de récupération, pouvant comprendre entre 50 et 300 personnes environ. Les occupants en sont régulièrement expulsés, se retrouvant dans la nécessité de perpétuellement négocier leur séjour avec un ensemble d'acteurs (propriétaires publics ou privés, municipalités, police). L'emplacement est trouvé « par essai et erreur », c'est-à-dire qu'un groupe sera chassé de commune en commune jusqu'à trouver une place de squat plus durable. Il en va ainsi d'un groupe de quelques centaines de Roms présents pour beaucoup depuis des années sur des terrains des environs de Pontoise.

Le fait que la plupart des bidonvilles de la région parisienne se trouvent dans des municipalités communistes ou socialistes des banlieues Nord et Est s'explique à la fois par la présence d'espaces disponibles dans ces communes (présence d'anciennes friches industrielles), et par la plus ou moins grande tolérance des municipalités et de leurs habitants à l'égard de ces occupants.

Occupant des espaces interstitiels dans la ville (sous les ponts d'autoroutes ou des échangeurs, sur d'anciennes friches industrielles, entre les voies ferrées), la quasitotalité des migrants sont victimes d'expulsions répétées, demandées par les propriétaires des lieux occupés, les municipalités ou les préfets ${ }^{13}$. Ajoutons que les conditions de vie dans ces espaces sont généralement extrêmement difficiles : la plupart sont boueux, infestés par les rats et les cafards, peu comprennent un point d'eau, généralement non potable, et les installations électriques de fortune se résument à de rares générateurs individuels. Les sanitaires y sont inexistants, les habitations sont perméables au froid et aux intempéries et les modalités de chauffage avec des bonbonnes de gaz ou des poêles de fortune représentent un danger d'incendie permanent, dont plusieurs migrants ont été victimes ${ }^{14}$.

Le « terrain » est une forme d'organisation à la fois très collective et très individualiste, ce qui peut être déconcertant pour les observateurs extérieurs et pour les ONG qui y interviennent. En effet, alors que ces rassemblements peuvent donner l'impression de former une communauté d'entraide, du fait de la promiscuité qui y règne et sachant que peu de faits restent du domaine privé, les comportements économiques y sont fondamentalement individualistes : l'argent est maintenu au sein de la famille et les vols entre migrants sont courants. Sur nombre de terrains, les regroupements se font par origine géographique (c'est le regroupement du réseau de migrants, même si celui-ci se recompose ou se désintègre avec les années), ce qui implique aussi

13 Un cas emblématique est celui de plusieurs groupes de Roms roumains qui sont, depuis plusieurs années régulièrement expulsés, et s'installent à quelques centaines de mètres plus loin, dans des champs et des forêts des communes de St. Ouen l'Aumône, Frépillon, Mérysur-Oise et Pierrelaye, en attente d'une nouvelle expulsion, dans le département du Val d'Oise (95).

14 Notamment le cas du décès de deux jeunes filles sur le bidonville rom de Surville à Lyon, en 2004, d'un jeune rom à Saint-Denis en 2007 ou d'un jeune enfant à Bobigny en 2009. Voir Romeurope (2008) et Bobigny : un enfant meurt dans l'incendie d'un camp rom, Le Parisien $23 / 05 / 2009$. 
le plus souvent un lien familial. Les relations internes de ces groupements peuvent alors ressembler à celles des villes ou villages d'origine. Mais sur la plupart des terrains, notamment les plus grands, les origines des personnes sont éclatées, ce qui signifie généralement une plus grande atomisation sociale.

Le regroupement joue un rôle important dans la vie des migrants, et donne un caractère particulier aux migrations des Roms ${ }^{15}$. En se constituant en problème collectif d'une municipalité, on encourt le risque de l'expulsion collective de ce terrain, mais on peut gagner un droit de séjour temporaire. Les avantages de ce type d'habitat résident aussi dans une certaine sécurité contre des attaques extérieures de la part de personnes qui voudraient s'y installer elles-mêmes ou dépouiller les migrants de leurs économies. Cette sécurité a ses limites. Selon des témoignages des migrants lors des visites de MDM, à plusieurs reprises, ces terrains ont été attaqués et rackettés de manière très violente par des bandes armées appartenant elles aussi au milieu des migrants roms roumains ou moldaves (notes de terrain, décembre 2005). Le collectif est également une sécurité car l'expulsion d'un groupe important présente, tant pour le propriétaire qui la demande que pour la police qui la met en œuvre, des difficultés pratiques et politiques.

\section{Le trompe-l'œil de la « communauté »}

Cependant, le regroupement a des inconvénients notables, dont les plus importants sont la promiscuité, l'insalubrité et l'insécurité. Le fait que ces terrains sont amenés à s'agrandir constamment est également un inconvénient : une négociation avec une municipalité qui s'engage à tolérer la présence des Roms ne pourra plus se mener lorsque le groupe passe d'une cinquantaine de personnes à deux cents habitants, comme il est arrivé à maintes reprises. Les terrains sont donc condamnés à être « cassés » (sparte, rou.), « explosés » (explodate, rou.) et leurs occupants à errer de commune en commune, nomades malgré eux, à la recherche d'un endroit où ils pourront rester quelques mois. Ce fut le cas par exemple entre 2004 et 2009 sur les territoires des communes de Saint-Denis, Saint-Ouen, Bagnolet, Pantin, Montreuil, où le dépassement d'une taille critique a entraîné une expulsion.

Parmi les migrants, un certain nombre, toujours de sexe masculin, s'approprient les «terrains » ou les squats occupés, devenant « chefs de terrain » (expression utilisée par les ONG), personnes dont la fonction oscille entre l'exploitation de la pauvreté et l'organisation de ces rassemblements de fortune. Ils exigent alors généralement un droit d'entrée, ou un loyer sur les différentes caravanes ou cabanes. En échange de ces taxes, le chef de terrain maintient un certain ordre, se charge de trouver un nouvel emplacement à la suite d'une expulsion, et assure les « relations au public » du groupe auprès des intervenants extérieurs (qui sont nombreux : police,

15 S'il existe de nombreux squats de migrants non-roms, je n'ai jamais rencontré en région parisienne de bidonville de migrants roumains ou bulgares non-roms. Des minorités de nonroms peuvent habiter dans des bidonvilles roms, mais il s'agit de situations rares et temporaires. 
ONG, municipalités, comités de soutien locaux). Cela signifie également que les chefs de terrain peuvent sélectionner les locataires potentiels, auxquels ils appliqueront des tarifs différenciés. Cette autorité a été décrite par ceux qui la subissaient dans des termes divers, allant de l'organisation au racket (cette dernière vision prédomine dans les entretiens). Dans les petits rassemblements qui sont presque toujours fondés autour d'une famille et d'une origine géographique commune, il n'existe généralement pas de chef de terrain.

Envers l'extérieur, les chefs de terrain se posent en représentants légitimes de la «communauté ». Les organisations qui interviennent sur le terrain ne saisissent pas toujours la nature de ce pouvoir et il arrive qu'elles considèrent ces personnes comme des sortes de « chefs de tribu », des patriarches bienveillants (Crochet, 2000). Cela relève en partie des contraintes intrinsèques de toute organisation qui, pour déléguer une tâche, ne peut traiter avec une pluralité d'individus mais a besoin de réduire le nombre des interlocuteurs. Cependant, le fait de considérer ces personnes comme les « responsables » ou les « représentants » des autres (vocabulaire souvent utilisé par les ONG) induit nombre d'effets pervers, contribuant à renforcer l'inégalité entre migrants.

\section{AMÉNAGER UNE SITUATION D'IMPASSE : L'ACTION DES AUTORITÉS LOCALES ET DES ONG}

La présence de groupes parfois larges de migrants illégaux et de squatters est devenue une question d'actualité pour nombre de municipalités, notamment celles de la « ceinture rouge » située au nord et à l'est de Paris, comme ce fut le cas dans des municipalités communistes (à Saint-Denis, Bagnolet, Achères), socialistes (Aubervilliers), vertes (L'Ile-Saint-Denis, Montreuil). Cependant dans les municipalités appartenant à la gauche la tolérance n'est nullement automatique ${ }^{16}$ et dépend largement des conjonctures municipales : le statut d'un terrain squatté peut déterminer la municipalité à s'abstenir de réclamer une expulsion, ou au contraire de la demander plus tôt ${ }^{17}$.

La tolérance vis-à-vis des groupes de Roms peut être pour la municipalité un enjeu face à la politique migratoire du gouvernement. Dans ces communes, les groupes de migrants doivent jongler entre visibilité et discrétion pour être tolérés aussi longtemps que possible ${ }^{18}$.

16 À propos du caractère répressif des politiques locales de sécurité selon la couleur politique des communes voir Mouhanna, 2005.

17 Le premier cas de figure est illustré par l'installation d'un groupe d'environ 500 Roms à Saint-Denis sur un espace occupé par le Cirque du Soleil en 2006, situation à l'origine d'un atermoiement et d'un projet de relogement de 80 personnes environ. En revanche, sur cette même commune, plusieurs groupes ont été expulsés de terrains sur lesquels des projets immobiliers ont été mis en place.

18 L'attitude des municipalités varie, entre le refus de l'installation, la majorité des communes, la tolérance plus ou moins grande de quelques-unes et, dans de rares cas, le soutien (ce fut le cas de celle d'Achères en 2003 lors de l'expulsion d'un bidonville rom, plus tard de celles de Lieusaint, St Denis, Aubervilliers ou Bagnolet). 
Dans les municipalités, minoritaires, où ils sont tolérés, ces migrants deviennent des enjeux de politique locale dans les luttes entre autorités municipales, formations politiques rivales, ONG, comités de soutien des habitants et police. Dans un contexte où la régularisation de ces personnes n'est pas envisageable (car celle-ci est de la compétence des préfets et, depuis la vague de régularisations de 1997, n'est plus accordée qu'au compte-gouttes et dans des cas particuliers (Cournil, 2008)), et où un permis de travail est quasi impossible à obtenir, les acteurs locaux doivent « bricoler » pour obtenir des améliorations partielles de la situation de ces personnes. Le plus souvent, ceci se résume à une garantie temporaire de la municipalité en ce qui concerne la nonexpulsion des terrains qu'elles occupent et à quelques efforts d'aménagement (installation de points d'eau, d'électricité, de toilettes, dératisation, ramassage des ordures). La situation juridique des migrants constitue cependant le principal obstacle à toute amélioration de leur condition : sans véritable droit au travail, ils restent condamnés à la mendicité ou à des activités illégales. La voie du logement social leur est également fermée.

Un facteur non négligeable dans ces aménagements urbanistiques et politiques est le marché de l'immobilier en Ile-de-France. Certaines communes anciennement ouvrières sont en passe de devenir la cible d'opérations immobilières et les anciens bâtiments industriels et les friches sont progressivement rachetés et rénovés. Ceci instaure une pression supplémentaire sur les municipalités pour expulser les migrants des terrains squattés, et repousse ceux-ci de plus en plus loin en banlieue (ce qui devient problématique lorsque leurs sources de revenu est la mendicité dans Paris intramuros). En 2006-2007 plusieurs terrains de Seine-Saint-Denis ont ainsi été évacués pour faire place à des chantiers et ont été remplacés à présent par des immeubles de bureaux ou des logements.

\section{Une action des autorités locales nécessairement limitée}

Depuis quelques années, des projets municipaux plus ambitieux ont vu le jour en région parisienne, dans lesquels une minorité des migrants est relogée (dans des caravanes ou des cabanes), et plus rarement régularisée, après des tractations entre certains maires et les préfets. Un événement comme une expulsion ou un incendie sur un terrain peut être le déclencheur d'un tel projet de relogement. Ces arrangements prennent la forme de conventions d'occupation signées entre municipalités et Roms. Il s'agit de décisions difficiles à prendre, car les municipalités prennent le risque de se retrouver en porte-à-faux entre les électeurs et les associations de défense des Roms. Elles comportent d'autres inconvénients, car la tolérance d'une municipalité tend à augmenter le nombre de migrants sur un terrain, les informations circulant très rapidement entre migrants.

Au mois de juin 2005, le Conseil régional d'Ile-de-France s'est à son tour engagé dans un projet intitulé «éradication des bidonvilles » et pour lequel un budget d'un million d'euros a été voté. Il s'agissait d'une aide à l'investissement pour les communes, communautés d'agglomérations ou conseils généraux qui présenteraient un projet permettant à un groupe de 80 à 100 personnes de quitter un bidonville pour s'installer dans un logement conforme à des normes minimales en termes d'habitat et d'équipements individuels. Cette initiative a conduit quelques municipalités (Auber- 
villiers, Saint-Denis, Bagnolet, Saint-Ouen) à proposer des projets de relogement pour environ 80 personnes entre 2005 et 2009. Un tel projet est en cours à Montreuil. Si cette mesure peut permettre d'améliorer la situation physique de quelques dizaines de personnes, elle ne fera que déplacer les centaines d'autres qui n’ont pas été sélectionnées. Chaque municipalité voit dans le « projet » la solution au problème de la présence des Roms et le départ des personnes excédentaires (Entretien avec un élu local, Bagnolet, juin 2006).

Cependant, ces opérations ont été décriées par nombre d'acteurs qui y ont participé en raison de leur caractère intrinsèquement discriminant : celles-ci étant nécessairement limitées quant au nombre de migrants qu'elles peuvent accueillir, du fait des moyens réduits des municipalités et de leur volonté de s'en tenir à des projets de petite taille considérés comme ayant plus de chance de réussite. Nombre d'ONG ont critiqué le fait de sélectionner des personnes en l'absence de critères clairs dans un groupe où activités licites et illicites sont souvent liées. Les critères de sélection ne peuvent être transparents car ils se résument le plus souvent à choisir les personnes les plus connues de la municipalité ou des différentes associations, qui n'ont qu'une connaissance partielle des migrants : les plus sympathiques, celles parlant mieux le français, ou celles qui parviennent à passer pour des « représentants ${ }^{19}$ de l'ensemble. Le choix de reloger « le groupe d'origine » s'avère lui aussi difficile car ce groupe est introuvable en raison des mouvements incessants et des liens familiaux entre anciens et nouveaux venus. Bien souvent, il s'agit là de pis-aller des politiques publiques, qui conduisent les municipalités à choisir un nombre limité de personnes parmi une population beaucoup plus nombreuse, au nom de la tradition d'accueil de la commune ou de la lutte contre la politique du gouvernement. Dans de telles conditions, rendre justice aux Roms d'un terrain est impossible, et le choix final s'expose à des récriminations incessantes.

De plus, la situation semi-légale de ces terrains protégés et la structure interne des groupes de migrants induisent parfois des effets pervers : détournement de ces opérations par des migrants extérieurs qui, par la menace, font partir les premiers occupants relogés et s'emparent de ces lieux pour en faire la base d'activités illicites. C'est ce qui a conduit certains projets à imposer des règles de vie particulièrement strictes : pas de visiteurs, couvre-feu. Les municipalités se retrouvent ainsi plus que jamais en situation de double-bind, entre les protestations des citoyens et la difficulté à reconnaître les limites de leur projet d' «intégration $»^{20}$. Ces opérations sont généralement sources de rupture et de conflits supplémentaires entre migrants, comme elles le sont entre les organisations en présence.

19 Le mot de « représentant » ou de « responsable » est souvent utilisé par les membres des municipalités ou des ONG pour désigner le chef de terrain, ce qui révèle les malentendus quant au véritable rôle de celui-ci.

20 Ce fut par exemple le cas de la convention signée par la municipalité de l'Ile-St-Denis avec un groupe de Roms en 2003, où le maire passa outre l'hostilité de la population pour aménager un terrain occupé par 75 personnes. Cependant, quelques mois plus tard ce nombre avait doublé et des cas de proxénétisme et d'exploitation de mineurs furent découverts après une arrestation collective dans le camp. Cette affaire fut exploitée par les médias et mit à rude épreuve la municipalité. En revanche, d'autres conventions, comme celle de Lieusaint en Seine-et-Marne ou d'Aubervilliers en Seine-Saint-Denis, purent déboucher sur un accord avec le préfet et des régularisations, qui permirent à beaucoup d'adultes de trouver un travail légal. Cependant seule une minorité des occupants d'origine du terrain fut incluse dans le projet. 


\section{Les dilemmes des ONG}

La cause des Roms, par excellence une «cause hérétique » pour citer la formule de Johanna Siméant au sujet des sans-papiers (Siméant, 1998 : 157), est également une cause paradoxale, qui peut s'accommoder de multiples penchants idéologiques (extrême-gauche, nationalisme ethniciste, bénévolat catholique etc.). Les principaux intéressés ne mesurent pas toujours les différents enjeux qui émergent dans la nébuleuse d'organisations en présence lors de ces opérations. Pour nombre d'ONG, les Roms sont décrits comme l'image même de la victime, ce qui leur permet de s'installer dans une posture dénonciatrice face aux pouvoirs publics, et encore davantage face aux pays d'origine des migrants (la Roumanie notamment), l'éloignement géographique accroissant la virulence de la dénonciation. Le thème des Roms facilite ces tendances car il s'agit d'une population qui en raison de son statut marginal mais aussi de sa culture cristallise aisément les fantasmes, allant de la figure du « bon sauvage » à celle des « véritables européens » de Günther Grass ${ }^{21}$.

Nous avons remarqué deux types de dilemmes auxquels sont confrontées les ONG.

Tout d'abord, celui des effets pervers de l'action des ONG : l'interaction entre pouvoirs publics et ONG peut avoir des effets inattendus ou non souhaités. Un exemple de ces dilemmes de la relation avec l'autorité publique est la scolarisation des enfants, dont MDM s'occupait avec la coopération d'autres associations comme ATD-Quart Monde. Les ONG visent les nombreux enfants qui passent des années sur les bidonvilles français dans un provisoire qui dure et qui ne sont en fin de compte jamais scolarisés. Cette activité souffre des contradictions inhérentes au fait d'attirer l'attention des institutions publiques sur l'existence d'une population en situation illégale : inscrire les enfants dans une école peut signifier ainsi conduire à l'expulsion de l'ensemble du terrain, comme cela est arrivé dans plus d'un cas. Ces enfants nouvellement scolarisés tendent à s'absenter, ce qui enclenche une procédure où l'école est légalement obligée de signaler ce fait à la DDASS et des représailles peuvent suivre pour les parents, voire pour l'ensemble du terrain.

Le deuxième est celui de la «participation » des intéressés aux programmes et actions mis en place pour eux. "Ce qui me désespère, c'est la passivité des gens. Ils se font expulser et au lieu de venir manifester avec nous devant la mairie, ils se sont éparpillés chacun dans leur coin » (entretien avec un bénévole, novembre 2006). Sur les terrains et squats, mais également sur les lieux où ils font la manche, les migrants se retrouvent dans une situation de constante négociation dans laquelle ils occupent la position du faible, que ce soit dans leurs relations avec les commerçants, la police, les municipalités, les ONG, situation d'une extrême précarité et instabilité. Ceci explique

21 «Les Roms sont ce que nous nous efforçons tous d'être : de véritables européens » (Grass, 1992). Le discours des municipalités qui s'engagent à reloger des migrants est en général moins unilatéral, plus réaliste que celui des ONG, car elles doivent faire face aux électeurs, qui ne sont pas tous exempts de préjugés racistes à l'égard des Roms. 
sans doute leur réticence à s'inscrire dans des actions véritablement « politiques », au sens d'actions collectives portant des revendications dans l'espace public, malgré les sollicitations des différentes organisations de défense des migrants ou des sans-papiers.

L'« approche participative » (Crochet, 2000), promue dans ces opérations de relogement comme dans le traitement de divers sujets comme la sanitation des bidonvilles, la scolarisation, l'accès à la santé, le « développement » sont différemment définis et leur sens change au fil des luttes de pouvoir entre associations. Parmi les intervenants de MDM, on mettait en avant la «participation des usagers » aux soins médicaux mais celle-ci, en pratique, est réduite à une acceptation d'un comportement « à l'occidentale ». Par exemple, dans le cadre d'une opération périnatalité, on s'attend à ce que les Roms reprennent à leur compte les pratiques de contraception qu'on tente de leur diffuser : «c'est pour ça qu'on fait autant de visites, c'est pour qu'ils participent aussi »(sage-femme, septembre 2004). Pour certaines municipalités engagées dans des projets de relogement, la «participation » signifiait que la sélection des bénéficiaires d'un programme serait effectuée par les chefs de terrain, vus comme « représentants » du groupe.

En réalité, une partie de ces limites de l'action des ONG est inévitable : en effet, il est difficile pour celles-ci, tout comme pour les autorités locales, de peser sur les véritables causes de l'exclusion des Roms et en premier lieu sur leur accès au travail. Dans cette situation, la plupart des ONG en sont réduites à des aménagements, une négociation permanente avec les pouvoirs publics. La défense des Roms reste une cause cloisonnée : si de nombreuses ONG qui soutiennent la cause des Roms soutiennent également les sans-papiers dans leur ensemble, les Roms sont vus comme à part, en raison de la discrimination spécifique qu'ils subissent et de la «culture rom » qui est la plupart du temps envisagée de façon essentialiste et exotisante. Ce cloisonnement encourage le développement d'un discours à la fois folkloriste et victimaire, oscillant entre identification avec les Roms et exotisation de ceux-ci.

\section{CONCLUSION}

Les migrations des Roms sont devenues des enjeux politiques externes et internes, ravivés dans les pays de départ à chaque atterrissage d'avion ramenant des nationaux renvoyés d'un pays occidental. Cependant, dans ce cas, le surcroît d'attention ne contribue pas à une amélioration durable de la situation des migrants. Au contraire, les différentes mesures prises à leur égard, la gestion souvent par défaut de cette population ont contribué à renforcer sa marginalisation.

Ces migrations sont à la fois une solution à court terme au problème de la pauvreté dans les pays d'origine et la cause d'une marginalisation d'un genre nouveau. Contrairement aux migrations de travail, les migrations des mendiants ne correspondent pas à une demande économique des pays-cibles. C'est une migration d' « inutiles », même si l'on peut arguer que le don au mendiant n'est pas sans contrepartie (symbolique, psychologique) pour le donneur. Peu qualifiés, faiblement insérés dans les autres réseaux de migrants est-européens, les Roms migrants ont 
cependant trouvé une place marginale dans la société française, tout en maintenant des liens sociaux et une certaine cohésion du groupe, de la famille, en évitant de tomber dans la clochardisation. Ils rappellent les vagabonds médiévaux, « placés en porte-àfaux dans cette conjoncture où la liberté leur advient comme une malédiction que l'on va essayer de retracer. Ils sont en situation de double bind, pris entre l'injonction de travailler et l'impossibilité de travailler sous les formes prescrites. » (Castel, 1999 : 139). Cette situation met en relief les limites d'une action pour leur défense qui rassemble avant tout acteurs locaux et ONG luttant avec des moyens limités contre des problèmes sur lesquels ils n'ont pas prise, et modifiant par là même la nature de leur action.

L'installation dans la mendicité, si elle est réelle, n'est pas la visée ultime de ces personnes. L'échec de leur migration, qui se voulait une migration de travail, est largement dû à leur capital social de départ : en cela, les migrations internationales reproduisent les inégalités socio-ethniques des pays d'origine. Zygmunt Bauman prend comme paradigme du monde post-moderne le «touriste » et le «vagabond », figures dialectiques et interdépendantes, tous deux exemples d'une fragmentation et d'un déracinement de la vie contemporaine (Bauman, 1998 : 77-103). Il s'agit là de formes de mobilité opposées mais qui quelquefois arrivent dans une situation de face-à-face, les mendiants interpellant les touristes sur l'inégalité sociale entre ces deux groupes. C'est cependant au monde des « touristes » que les « vagabonds » mesurent leur situation. Cela montre, en creux, que même pour ses citoyens les plus pauvres, l'Europe reste cette norme implicite de «bonne vie », et que c'est l'expérience des migrations qui établit et renforce cette norme.

\section{Références bibliographiques}

BAUMAN Zygmunt (1998) Globalization, the Human Consequences, Columbia, New York, University Press, $136 \mathrm{p}$.

BIGO Didier, GUILD Elspeth (2005) Policing at a distance: Schengen Visa Policies, in Didier Bigo, Elspeth Guild, Controlling Frontiers, Aldershot, Ashgate, pp. 233-263.

CASTEL Robert (1999) Les métamorphoses de la question sociale, Paris, Gallimard, 813 p.

CASTLE-KARENOVA Mita (2003) Round and Round the Roundabout: Czech Roma and the Vicious Circle of Asylum-Seeking, Nationalities Papers, $\mathrm{n}^{\circ}$ 1, pp. 13-25.

COURNIL Christel (2008) La circulaire du 13 juin 2006, une régularisation sélective au service d'une politique : à propos de la régularisation des parents d'enfants scolarisés, Revue de la recherche juridique, droit prospectif, $\mathrm{n}^{\circ} 123$, pp. 1495-1516.

CROCHET Soisick (2000) Cet obscur objet du désir : la participation communautaire, in Brauman Rony, Utopies sanitaires, Paris, Le Pommier, pp. 47-76.

CROWE David (2007) A History of the Gypsies of Eastern Europe and Russia, New York, Palgrave Macmillan, $416 \mathrm{p}$.

COMMISSION EUROPÉENNE (2004) La Situation des Rom dans une Union européenne élargie, http://ec.europa.eu/employment_social/publications/2005/ke6204389_fr.pdf, dernier accès : août 2008, 70 p.

DAMON Julien (1998) Vagabondage et mendicité, Paris, Flammarion, 125 p. 
DIMINESCU Dana et LAGRAVE Rose-Marie (1999) Faire une saison. Pour une anthropologie des migrations roumaines en France. Le cas du pays d'Oas, Migrations Études, n ${ }^{\circ}$, pp. 114.

FIDH [Fédération Internationale des Ligues des Droits de l'homme], MDM [MÉDECINS DU MONDE] (2003) Mission d'évaluation en Roumanie, http://www.medecinsdumonde.org/fr/content/download/1918/14778/file/roumanie_MdM_FIDH.pdf dernier accès août 2008.

GRASS Günther (1992) Losses, Granta, n 42, pp. 97-108.

GUGLIEMO Rachel (2004) Human Rights in the Accession Process: Roma and Muslims in an Enlarging Europe, Minority Protection and the Enlarged European Union: The Way Forward, Local Government and Public Service Reform Initiative, Open Society Institute, pp. 37-59.

MASSEY Douglas (1988) Economic Development and Migration in Comparative Perspective, Population and Development Review, $\mathrm{n}^{\circ}$ 3, pp. 375-397.

MOUHANNA Christian (2005) Coproduction, cohérence ou concurrence ? Réflexions sur la coopération élus-policiers en matière de sécurité, in Jérôme Ferret, Christian Mouhanna, Peur sur les villes, Paris, Presses universitaires de France, 229 p.

NACU Alexandra (2006) La construction sociale de la pauvreté en Roumanie et en Bulgarie après 1989, thèse de doctorat en sciences politiques soutenue à Sciences-Po, Paris.

POTOT Swanie (2007) Vivre à l'Est, travailler à l'Ouest : les routes roumaines de l'Europe, Paris, L'Harmattan, $227 \mathrm{p}$.

ROMEUROPE (2008) Rapport 2007-2008, Paris, 172 p.

SIMEANT Johanna (1998) La cause des sans-papiers, Paris, Presses de la FNSP, 504 p.

STEWART Michael (1997) The time of the Gypsies, Boulder, Westview Press, 302 p.

VASECKA Imrich and VASECKA Michal (2003) Recent Romani Migration from Slovakia to EU Member States: Romani Reaction to Discrimination or Romani Ethno-Tourism?, Nationalities Papers, ${ }^{\circ}$ 1, pp. 27-45. 


\title{
Les Roms migrants en région parisienne : les dispositifs d'une marginalisation
}

\begin{abstract}
Alexandra NACU
Cet article s'intéresse aux Roms est-européens installés en banlieue parisienne et aux formes d'intervention institutionnelle (pouvoirs publics et $\mathrm{ONG}$ ) à leur endroit. En analysant ce phénomène à différentes échelles (européenne et locale), l'auteure montre comment ce groupe, sédentaire à l'origine, est maintenu avec le concours de l'action des pouvoirs publics dans un nomadisme forcé, perpétuant et même renforçant ainsi sa marginalisation.
\end{abstract}

\section{Roma Migrants in the Paris Suburbs: Mechanisms of Marginalization}

\section{Alexandra NACU}

This article focuses on Eastern European Roma living in the Parisian suburbs and to various form of institutional intervention regarding them. The author analyse the interaction between multiple scales of actors involved in this management as well as the kind of mobilization induced. At both levels, European and local, one can observe numerous relegation devices that, without necessarily being intentional or institutionalized, concur in producing this population's marginalization.

\section{Los migrantes Roma en la region de Paris: los mecanismos de la marginalizacion}

\section{Alexandra NACU}

El articulo analiza la situacion de los Roma (Gitanos) originarios de Europa de Este que viven en las suburbias de Paris, y sus interacciones con varias instituciones. A los ambos niveles, europeo y local, existen varios mecanismos que, muchas veces de manera involuntaria, resultan en la exclusion de los migrantes. 